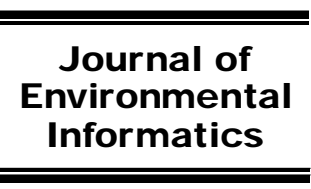

www.iseis.org/jei

\title{
A Fuzzy Evacuation Management Model Oriented Toward the Mitigation of Emissions
}

\author{
Q. $\operatorname{Tan}^{1,2}$, G. H. Huang ${ }^{3, *}$, and Y. P. Cai ${ }^{4,2}$ \\ ${ }^{I}$ College of Water Resources \& Civil Engineering, China Agricultural University, Beijing 100083, China \\ ${ }^{2}$ Institute for Energy, Environment and Sustainable Communities, University of Regina, Regina, Saskatchewan S4S $7 H 9$, Canada \\ ${ }^{3}$ Faculty of Engineering and Applied Science, University of Regina, Regina, Saskatchewan S4S 0A2, Canada \\ ${ }^{4}$ State Key Laboratory of Water Environment Simulation, School of Environment, Beijing Normal University, Beijing 100875, China
}

Received 12 April 2012; revised 20 August 2013; accepted 10 December 2013; published online 28 August 2014

\begin{abstract}
Although the transportation sector is a major contributor to urban air pollution and global climate change due to its substantial energy consumptions, previous studies for evacuation practices in this sector seldom took environmental consequences into account. As an attempt in event-related evacuation planning under uncertainty, this study proposed an emission-mitigation-oriented fuzzy evacuation management (emoFEM) model. Comprehensive considerations over system efficiency, environmental protection, economic cost and resource availability were incorporated within a general modeling formulation to facilitate evacuation management in a systematic and compromise manner. Vague and ambiguous information embedded within evacuation problems could be quantified and directly communicated into the optimization process, greatly improving conventional tools for evacuation management under uncertainty. The proposed emoFEM model was then applied to a hypothetic but representative case. Useful solutions were generated, which could help identify timely, safe and cost-effective evacuation schemes without significant disturbances over normal municipal traffic and environmental quality. The advantages of emoFEM were further revealed through comparing its solutions with those from its deterministic counterpart.
\end{abstract}

Keywords: evacuation management, emission mitigation, transportation, fuzziness, uncertainty

\section{Introduction}

The transportation sector accounts for about $25 \%$ of total commercial energy consumed and $50 \%$ of total oil produced worldwide, representing a major contributor to urban air pollution and global climate change (Gorham, 2002; Yoshida and Matsuhashi, 2009; Birant, 2011). There is thus a growing awareness of its role in the efforts for mitigating emissions of air pollutants and greenhouse gases. Among various transporttation activities, event-related evacuation services have been growing considerably along with economic growth and the trend toward urbanization and globalization. Event-related evacuation can be defined as the mass movement of evacuees from an event venue to a number of destinations through public transport in response to a large-scale event instead of a danger or threat. It causes intensified transportation demands as well as increased environmental burdens, requiring decision makers to balance the objective of higher evacuation efficiency against those of lower economic cost and reduced environmental impact. Meanwhile, evacuation problems are fur-

${ }^{*}$ Corresponding author. Tel.: +1 306 5854095; fax: +1 3065854855.

E-mail address: gordon.huang@uregina.ca (G. H. Huang).

ISSN: 1726-2135 print/1684-8799 online

(C) 2014 ISEIS All rights reserved. doi:10.3808/jei.201400272 ther complicated by a variety of uncertainties, necessitating the development of scientific tools for supporting relevant decision making under uncertainty.

Interests in evacuation modeling began in the 1970s (Urbanik, 1978; Wilmot and Mei, 2004). Over the past decades, a number of studies were conducted. For example, Cova and Church (1997) employed an integer programming model to assess vulnerability of evacuation in communities. Hobeika and Kim (1998) proposed a mass evacuation computer program (MASSVAC 4.0) to simulate traffic movements within an evacuation process based on a user equilibrium assignment algorithm. Jha et al. (2004) formulated a microscopic simulation model (MITSIMLab) to model traffic operations, and presented a laboratory-like setup to evaluate a large range of evacuation scenarios. Liu et al. (2006) developed a two-level integrated optimization model for generating candidate sets of optimal evacuation plans that serve as inputs for simulationbased evacuation systems. Yi and Ozdamar (2007) developed a mixed integer multi-commodity network flow model for coordinating logistics support and evacuation operations. Lin et al. (2008) proposed a multi-stage time-varying quickest flow model based on a time-varying dynamic network to optimize the evacuation routes and schedule under an emergency situation. Xie et al. (2010) discussed a dynamic evacuation network optimization problem based on a bi-level model that incorporates land reversal and crossing elimination strategies. 
However, previous studies suffered from two major limitations: (i) most of them focused on emergency evacuation induced by extreme events (e.g., hurricanes, fires, and nuclear power plant accidents), where minimized evacuation time is the sole objective. Few of them were pertinent to event-related evacuation management which is common in practice and requires comprehensive considerations over many factors; and (ii) inherent uncertainties existing in many components of evacuation management systems were largely overlooked by previous studies.

Uncertainty plays an important role in evacuation management problems. Although stochastic programming could be effective for dealing with uncertainties that are expressed as probability distribution functions, such exact information for many parameters, such as operating costs of vehicles, are usually unavailable in practice. The process of evacuation planning is usually fraught with fuzziness, which is linguistic in nature and stems from imprecise, vague and ambiguous information (Huang et al., 1993, 1996; Huang and Chang, 2003; Cai et al., 2009, 2011b, 2011c; Tan et al., 2010b, 2011a, 2011b; Gunalay et al., 2012). Fuzzy mathematical programming (FMP) based on fuzzy sets theory is thus viewed as an attractive alternative to the stochastic one in the absence of sufficient data to describe the statistics of uncertain parameters. FMP approaches can be classified into two categories: possibilistic programming and flexibility programming. In possibilistic programming, uncertain parameters are modeled as fuzzy sets. Fuzzy robust linear programming (FRLP), as a typical possiblilistic programming approach, delimits an uncertain decision space by specifying uncertainties through dimensional enlargement of the original fuzzy constraints (Luhandjula and Gupta, 1996; Liu et al., 2003; Cai et al., 2011a). Although FRLP is effective for reflecting fuzzy coefficients in both left- and right-hand sides of the model's constraints, it is limited to deterministic coefficients in the objective function (Tan et al., 2010a, 2013; Dong et al., 2014). Different from and complementary to possibilistic programming, flexible fuzzy linear programming (FLP) is capable of tackling fuzziness in the model's goals and stipulations (Dubois and Prade, 1999; Huang et al., 2001). There have been few studies in the literature involving the use of FMP models for tackling evacuation management problems. Introduction of FRLP and FLP into evacuation management would prospectively facilitate relevant decision making under a fuzzy environment.

This study aims at proposing an emission-mitigationoriented fuzzy evacuation management (emoFEM) model for supporting integrated event-related evacuation planning under a fuzzy environment. This model will provide scientific bases for identifying vehicle allocation plans and routing strategies through reflecting the peculiarities of event-related evacuation activities. Considerations over not only evacuation efficiency, but also environmental protection and economic cost, will be integrated into a general modeling formulation. The emoFEM model will also be capable of quantifying vague and ambiguous information that ubiquitously exist in evacuation management systems. The proposed model will then be applied to a hypothetic but representative case of event-related evacuation management for demonstrating its applicability.

\section{Modeling Formulation}

Evacuation demands induced by events are normally predictable, enabling proactive planning and analysis. Evacuation schemes are highly dependent on the venues, scales and schedules of events, requiring site-specific plans. Evacuees mostly originate from a single venue and are to be sent to several pre- specified destinations. Unlike emergency-induced evacuation with the minimized evacuation time being the top and sole objective, event-related evacuation management requires a comprehensive consideration over a broad spectrum of issues such as system efficiency, economic cost, and environmental restrictions. The above characteristics distinguish event-related evacuation processes from emergency-induced ones. Scientific planning for routing strategies and vehicle allocation is the major concern, which primarily relies on efficient utilization of transportation resources and services (Chiu et al., 2007; Wu et al., 2009). Recent advancements in sensor technologies have facilitated the acquisition of a wide range of traffic information, from tracking vehicles presence, volume and speed, to detecting vehicle density or travel time (Hamza-Lup et al., 2005). Short-term traffic information could be obtained from a number of sources, such as sensor acquisitions, historical records, and expert judgments. Considering an evacuation system wherein decision makers are respon- sible for evacuating a large number of people from an origin to multiple destinations through public transit after a large-scale event, a deterministic emission-mitigation-orientted evacuation management (emoEM) model can be formulated as below.

\subsection{Objective Function}

As the top priority of evacuation management, minimized total evacuation time is the objective of the proposed model. Generally, evacuation time is the summation of total time needed for all of the evacuees to reach their correspondding destinations, which includes running time on links as well as waiting time at traffic lights and toll stations:

$$
\begin{aligned}
\min t= & \sum_{s}^{S} \sum_{a}^{A_{s}} \sum_{i}^{I} x_{\text {sai }} p_{i} \sum_{m \in s a} t_{m}+\sum_{s}^{S} \sum_{a}^{A_{s}} \sum_{i}^{I} x_{s a i} p_{i} \sum_{k \in s a}^{K_{s a}} L t_{k} \\
& +\sum_{s}^{S} \sum_{a}^{A_{s}} \sum_{i}^{I} x_{s a i} p_{i} \sum_{r \in s a}^{R_{s a}} T t_{r}
\end{aligned}
$$

where $S$ is the index for destinations; $A_{s}$ is the index for paths from evacuation origin to destination $s$; $I$ is the index for vehicle types; $x_{\text {sai }}$ is number of $i$-type vehicles designated to path $s a$ to reach destination $s$ (veh, decision variable); $p_{i}$ is the passenger loading capacity of an $i$-type vehicle (persons/veh); $t_{m}$ is the travel time on link $m, t_{m}=l_{m} / v_{m}(\mathrm{hr}) ; K_{s a}$ is the index for nodes with traffic lights on path $s a ; L t_{k}$ is the average delay time at node $k$ due to a traffic light (hr); $R_{s a}$ is the index for toll 
stations on path $s a$; $T t_{r}$ is the average delay time at toll station $r$ (hr).

\subsection{Constraints}

The constraints include emission and cost restrictions, as well as the relationships among the decision variables and the evacuation demand/management conditions. Six types of constraints are included in the model. They are emission, cost, vehicle-availability, passenger-balance, road-capacity, and technical constraints.

\subsubsection{Allowable Emission Constraint}

Vehicles burning gasoline and diesel fuel are a major source of air pollutants and greenhouse gases (Singer and Harley, 1996; Gorham, 2002). Control of emissions from vehicular traffic during evacuation activities should thus be one of the most important elements of integrated evacuation management. Among those emitted pollutants from traffic, carbon monoxide (CO) is capturing more and more attention. This is mainly attributed to two facts: (i) traffic is the main contributor of $\mathrm{CO}$ emissions in urban areas. For example, traffic is responsible for $99 \%$ of emitted CO in London (Read, 1994). (ii) CO is high toxic, posing serious threats to human health and the environment (Kongtip et al., 2006; Gokhale and Khare 2007). Thus, CO has been frequently used as a predominant indicator of pollutant emissions in many transportation-related studies (Read, 1994; Wu et al., 2009). The related emission standards for $\mathrm{CO}$ can be obtained from the regulations of municipal authorities. In this study, $\mathrm{CO}$ emissions from evacuation activities are considered to fall into two categories: running and idle. The former is related to the numbers of vehicles, lengths of links, and emission factors of vehicles under running condition which are functions of speed on given links ( $\mathrm{Li}, 2001)$. The latter mainly depends on the numbers of vehicles, the average delay time at traffic lights, and the idle emission factors which can be obtained from test data of a large number of vehicles (Li, 2001). The emission constraint is thus presented as follows:

$$
\begin{aligned}
& \sum_{s}^{S} \sum_{a}^{A_{s}} \sum_{i}^{I} x_{s a i} \sum_{m \in s a} l_{m} E C_{m i} \\
& +\sum_{s}^{S} \sum_{a}^{A_{s}} \sum_{i}^{I} x_{s a i}\left(\sum_{k \in s a}^{K_{s a}} L t_{k}\right) E I C_{i} \\
& +\sum_{s}^{S} \sum_{a}^{A_{s}} \sum_{i}^{I} x_{s a i}\left(\sum_{r \in s a}^{R_{s a}} T t_{r}\right) E I C_{i} \leq E B
\end{aligned}
$$

where $E C_{m i}$ is the $\mathrm{CO}$ emission factor of an $i$-type vehicle on link $m$ under running condition $(\mathrm{g} / \mathrm{veh} \cdot \mathrm{km}), E C_{m i}=\alpha_{i}+\beta_{i} v_{m}$ $+\gamma_{i} v_{m}^{2} ; \alpha_{i}, \beta_{i}, \gamma_{i}$ are the coefficients of the emission factor for an $i$-type vehicle under running condition; $v_{m}$ is the average velocity on link $m(\mathrm{~km} / \mathrm{hr}) ; E I C_{i}$ is the CO emission factor of an $i$-type vehicle under idle condition ( $\mathrm{g} / \mathrm{veh} \cdot \mathrm{hr}$ ), which can be estimated based on a number of test data; $E B$ is the total allowable emission amount $(\mathrm{g})$.

\subsubsection{Operating Cost Constraint}

Operating costs mainly include expenses for consumption of fuel, depreciation of vehicles, and wages of drivers. They are typically dependent on the distances of driving and the types of vehicles. The operating cost constraint is formulated as follows:

$\sum_{s}^{S} \sum_{a}^{A_{s}} \sum_{i}^{I} x_{s a i}\left(\sum_{m \in s a} l_{m}\right) F_{i} \leq F B$

where $l_{m}$ is the length of link $m(\mathrm{~km}) ; F_{i}$ is the unit operating cost of an $i$-type vehicle $(\$ / \mathrm{km}) ; F B$ is the total allowable costs $(\$)$.

\subsubsection{Vehicle Availability Constraints}

Generally, vehicles for evacuation can be classified into several types according to their varied passenger-loading capacities. The mass balance of vehicles is:

$\sum_{s}^{S} \sum_{a}^{A_{s}} x_{s a i} x \leq N_{i} \quad \forall i$

where $N_{i}$ is the number of available $i$-type vehicles (veh).

\subsubsection{Passenger Balance Constraints}

Evacuees can be grouped according to their destinations. The mass balance for evacuees is written as:

$\sum_{a}^{A_{s}} \sum_{i}^{I} x_{s a i} p_{i} \geq D_{s} \quad \forall s$

where $D_{s}$ is the number of evacuees whose destination is $s$.

\subsubsection{Road Capacity Constraints}

Intensive transportation demands (in terms of both space and time) from evacuation activities, combined with normal traffic in urban areas which is probably exposed to severe congestions, pose a major challenge to evacuation planners. The vehicles designated for evacuation should not lead to much disturbance over normal municipal traffic. The constraints for road capacities can be written as:

$$
\sum_{m \in s a}^{A_{s}} \sum_{s}^{S} \sum_{i}^{I} e_{i} x_{s a i}+M X_{m} \leq V M_{m} \quad \forall m
$$

where $e_{i}$ is the equivalent conversion coefficient of an $i$-type vehicle; $M X_{m}$ is the existing traffic flow on link $m$ (veh); $V M_{m}$ is the maximum capacity of link $m$ (veh);

\subsubsection{Non-Negativity and Technical Constraints}

The non-negativity and technical constraints include: 
$x_{\text {sai }}\left\{\begin{array}{l}\geq 0 \\ =\text { integer }\end{array} \forall s, a, i\right.$

Although model (1) presents general formulas for an event-related evacuation management model where a number of factors are considered, it is not sufficient to model such a complex system due to the inherent uncertainties in decision makers' preferences and values of parameters. Firstly, the management goal of decision makers may be fuzzy. For example, "the total evacuation time for evacuating all of the event participants to their destinations should be less than $1000 \mathrm{hrs}$, preferably no more than 800 hrs". Secondly, the stipulations of some constraints can be relaxed within certain tolerance intervals. For example, "the maximum allowable amount of pollutants emitted from evacuation vehicles would be 8,500 to $12,000 \mathrm{~g}$ ". Thirdly, many parameters are likely to be estimated by experts as triangular fuzzy sets with a most possible value instead of deterministic numbers. For example, "the unit operating cost for an intermediate passenger car is approximately $\$ 0.5 / \mathrm{km} "$. To address such uncertainties, an emoFEM model is established as follows

$$
\begin{aligned}
\min t= & \sum_{s}^{S} \sum_{a}^{A_{s}} \sum_{i}^{I} x_{s a i} p_{i} \sum_{m \in s a} t_{m}+\sum_{s}^{S} \sum_{a}^{A_{s}} \sum_{i}^{I} x_{s a i} p_{i} \sum_{k \in s a}^{K_{s a}} L t_{k} \\
& +\sum_{s}^{S} \sum_{a}^{A_{s}} \sum_{i}^{I} x_{s a i} p_{i} \sum_{r \in s a}^{R_{s a}} T t_{r}
\end{aligned}
$$

subject to:

$$
\begin{aligned}
& \sum_{s}^{S} \sum_{a}^{A_{s}} \sum_{i}^{I} x_{s a i} \sum_{m \in s a} l_{m} E C_{m i}+\sum_{s}^{S} \sum_{a}^{A_{s}} \sum_{i}^{I} x_{s a i}\left(\sum_{k \in s a}^{K_{s a}} L t_{k}\right) E I C_{i} \\
& +\sum_{s}^{S} \sum_{a}^{A_{s}} \sum_{i}^{I} x_{s a i}\left(\sum_{r \in s a}^{R_{s a}} T t_{r}\right) E I C_{i} \leq E B \\
& \sum_{s}^{S} \sum_{a}^{A_{s}} \sum_{i}^{I} x_{\text {sai }}\left(\sum_{m \in s a} l_{m}\right) F_{\sim} \leq F B_{\sim} \\
& \sum_{s}^{S} \sum_{a}^{A_{s}} x_{s a i} \leq N_{i} \quad \forall i \\
& \sum_{a}^{A_{s}} \sum_{i}^{I} x_{s a i} p_{i} \geq D_{s} \quad \forall s \\
& \sum_{m \in s a}^{A_{s}} \sum_{s}^{S} \sum_{i}^{I} e_{i} x_{s a i}+M X_{m} \leq V M_{m} \quad \forall m \\
& x_{s a i} \geq 0 \\
& =\text { integer } \quad \forall s, a, i
\end{aligned}
$$

where $\bar{\approx}$ and $\leqq$ denote fuzzy equality and inequality, respecttively; $F$ and $F B$ are triangular fuzzy sets.
Based on the principle of FLP, a decision in a fuzzy environment is defined as the intersection of membership functions corresponding to fuzzy objective and constraints (Zimmermann, 2001). Assume that there are $n$ fuzzy goals $\left(G_{1}\right.$, $\left.G_{2} \ldots, G_{n}\right)$ and $m$ fuzzy constraints $\left(C_{1}, C_{2}, \ldots, C_{m}\right)$ in a space of alternatives $(X)$. The resulting fuzzy decision set $(D)$ will then be an intersection of the given goals and constraints as follows:

$D=G_{1} \cap G_{2} \cap \cdots \cap G_{n} \cap C_{1} \cap C_{2} \cap \cdots \cap C_{m}$

Correspondingly:

$\mu_{D}=\operatorname{Min}\left\{\mu_{G 1}, \mu_{G 2}, \cdots, \mu_{G n}, \mu_{C 1}, \mu_{C 2}, \cdots, \mu_{C m}\right\}$

where $\mu_{D}, \mu_{G}$, and $\mu_{C}$ denote membership functions of fuzzy decision, fuzzy goals, and fuzzy constraints, respectively. Let each of the fuzzy goals and constraints be represented by a fuzzy set with a membership function $\mu_{i}(X)(i=1,2, \cdots$, $m+n) ; \mu_{i}(X)$ should be 0 if the $i$ th aspired objective or constraint is violated, or be 1 if it is totally satisfied. Thus, the membership function of the fuzzy decision is:

$\mu_{D}(X)=\operatorname{Min}\left\{\mu_{i}(X) \mid i=1,2, \ldots, m+n\right\}$

Introduce an independent variable $\lambda=\mu_{D}(X) . \lambda$ can reflect the flexibility in the constraints and fuzziness in the objectives, corresponding to the degree of overall satisfaction for the constraints and objectives (Zimmermann, 2001; Dubois and Prade, 1999; Huang et al., 2001). A desired decision is thus the one with the highest $\lambda$ value:

$$
\operatorname{Max} \lambda=\operatorname{Max} \mu_{D}(X)=\operatorname{Max} \operatorname{Min}\left\{\mu_{i}(X) \mid i=1,2, \ldots, m+n\right\}
$$

Based on the concept of level set (fuzzy $\alpha$-cut), the $\alpha$ level set of $F_{\sim i}$ is a crisp subset of $X$ which can be defined as follows:

$$
F_{\sim \alpha \alpha}=\left\{x \mid \mu_{F_{i}}(x) \geq \alpha \text { and } x \in X\right\}
$$

Under a $\alpha$-cut level $\alpha_{w}\left(\alpha_{w} \in[0,1], w=1,2, \ldots, u\right)$, the superior and inferior limit values of $\left(F_{\sim i}\right)_{\alpha_{w}}$ are denoted as $\bar{F}_{i}^{w}$ and $F_{i}^{w}$, respectively. Likewise, for fuzzy set $F B$, we can also get an interval $\left[\underline{F B}^{w}, \overline{F B}^{w}\right]$ under each $\alpha_{w}(\tilde{w}=1,2, \ldots, u)$ (Soyster, 1973; Leung, 1988; Luhandjula and Gupta, 1996; Inuiguchi and Sakawa, 1998). In FRLP, the possible region of occurrence for the left-land side of each constraint containing fuzzy coefficients is considered to be contained in the satisfactory or tolerable region as defined by the corresponding right-hand side (Inuiguchi and Sakawa, 1998; Liu et al., 2003). Thus, each constraint containing fuzzy coefficients can be replaced by $2 u$ inequalities, in which $u$ denotes $u$ levels of $\alpha$-cut. The decision space for the problem can then be delimited. 
Through incorporating FLP and FRLP into the framework of emoEM, model (2) can be transformed into the following problem:

$\operatorname{Max} \lambda$

subject to:

$$
\begin{aligned}
& \sum_{s}^{S} \sum_{a}^{A_{s}} \sum_{i}^{I} x_{s a i} p_{i} \sum_{m \in s a} t_{m}+\sum_{s}^{S} \sum_{a}^{A_{s}} \sum_{i}^{I} x_{s a i} p_{i} \sum_{k \in s a}^{K_{s a}} L t_{k} \\
& +\sum_{s}^{S} \sum_{a}^{A_{s}} \sum_{i}^{I} x_{s a i} p_{i} \sum_{r \in s a}^{R_{s a}} T t_{r} \leq f_{o p t 1}^{+}-\lambda\left[f_{o p t 1}^{+}-f_{o p t 1}^{-}\right] \\
& \sum_{s}^{S} \sum_{a}^{A_{s}} \sum_{i}^{I} x_{s a i} \sum_{m \in s a} l_{m} E C_{m i}+\sum_{s}^{S} \sum_{a}^{A_{s}} \sum_{i}^{I} x_{s a i}\left(\sum_{k \in s a}^{K_{s a}} L t_{k}\right) E I C_{i} \\
& +\sum_{s}^{S} \sum_{a}^{A_{s}} \sum_{i}^{I} x_{s a i}\left(\sum_{r \in s a}^{R_{s a}} T t_{r}\right) E I C_{i} \leq E B^{+}-\lambda\left[E B^{+}-E B^{-}\right] \\
& \sum_{s}^{S} \sum_{a}^{A_{s}} \sum_{i}^{I} x_{s a i}\left(\sum_{m \in s a} l_{m}\right) \bar{F}_{i}^{w} \leq \overline{F B}^{w} \quad w=1,2, \cdots, u \\
& \sum_{s}^{S} \sum_{a}^{A_{s}} \sum_{i}^{I} x_{s a i}\left(\sum_{m \in s a} l_{m}\right) \underline{F}_{i}^{w} \geq \underline{F B}^{w}
\end{aligned}
$$$$
\sum_{s}^{S} \sum_{a}^{A_{s}} x_{s a i} \leq N_{i} \quad \forall i
$$$$
\sum_{a}^{A_{s}} \sum_{i}^{I} x_{s a i} p_{i} \geq D_{s} \quad \forall s
$$$$
\sum_{m \in s a}^{A_{s}} \sum_{s}^{S} \sum_{i}^{I} e_{i} x_{s a i}+M X_{m} \leq V M_{m} \quad \forall m
$$

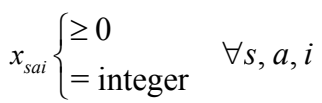

$0 \leq \lambda \leq 1$

\section{Case Study}

An evacuation network built upon representative data from relevant literature and typical Chinese cities (e.g., the City of Wuhan) is studied to explore the practicability of the proposed emoFEM model. The study network consists of one origin, three destinations (D1 to D3), two traffic lights (Lt1 and Lt2), one toll station (T1), and eight links connecting the nodes (L1 to L8) (Wu et al., 2009). The evacuation planners are responsible for evacuating the event participants through public transit from the evacuation origin to the three destinations scattered in a city. For each destination, a number of alternative paths exist. Each path, which is comprised of se- veral links, exhibits an individual geometric configuration. In detail, to reach destinations 1, 2 and 3, there are two, three and two alternative paths, respectively. Evacuating the evacuees to their target destinations through these paths requires a systematic consideration of the flow and capacity of each path and its constituent links (Chiu et al., 2007). Lengths, average velocities, maximum capacities, and existing traffic flows of links are presented in Table 1.

Table 1. Lengths, Average Velocities, Existing Traffic Flows and Maximum Capacities of the Links

\begin{tabular}{lllll}
\hline & Length & $\begin{array}{l}\text { Average } \\
\text { velocity }\end{array}$ & $\begin{array}{l}\text { Existing } \\
\text { traffic flow } \\
\mathrm{MX}_{\mathrm{m}}(\mathrm{veh})\end{array}$ & $\begin{array}{l}\text { Maximum } \\
\text { capacity } \\
\mathrm{VM}_{\mathrm{m}}(\mathrm{veh})\end{array}$ \\
\hline L1 & 35 & 70 & 1800 & 2350 \\
L2 & 8 & 27 & 450 & 680 \\
L3 & 12 & 36 & 990 & 1050 \\
L4 & 6 & 20 & 180 & 440 \\
L5 & 9 & 45 & 540 & 710 \\
L6 & 4 & 30 & 265 & 510 \\
L7 & 2.1 & 27 & 90 & 300 \\
L8 & 2.3 & 36 & 220 & 400 \\
\hline
\end{tabular}

Table 2. Numbers, Passenger Loading Capacities and Equivalent Conversion Coefficients of the Vehicles

\begin{tabular}{llll}
\hline & $\begin{array}{l}\text { Available } \\
\text { number } \mathrm{N}_{\mathrm{i}} \\
(\mathrm{veh})\end{array}$ & $\begin{array}{l}\text { Passenger loading } \\
\text { capacity } \mathrm{p}_{\mathrm{i}} \\
\text { (persons } / \mathrm{veh})\end{array}$ & $\begin{array}{l}\text { Equivalent } \\
\text { conversion } \\
\text { coefficient } \mathrm{e}_{\mathrm{i}}\end{array}$ \\
\hline Light-duty & 150 & 18 & 1.2 \\
Medium-duty & 90 & 27 & 2.0 \\
Heavy-duty & 125 & 54 & 3.0 \\
\hline
\end{tabular}

Table 3. Emission Factors of the Vehicles under Running and Idle Conditions

\begin{tabular}{lll}
\hline & $\begin{array}{l}\text { Running condition }\left(\mathrm{EC}_{\mathrm{mi}},\right. \\
\mathrm{g} / \mathrm{veh} \cdot \mathrm{km})\end{array}$ & $\begin{array}{l}\text { Idle condition }\left(\mathrm{EIC}_{\mathrm{i}},\right. \\
\mathrm{g} / \mathrm{veh} . \mathrm{hr})\end{array}$ \\
\hline Light-duty & $\begin{array}{l}81.8760-1.8551 \mathrm{v}_{\mathrm{m}}+ \\
0.0123 \mathrm{v}_{\mathrm{m}}^{2}\end{array}$ & 379 \\
& $\begin{array}{l}138.9050-3.1472 \mathrm{v}_{\mathrm{m}}+ \\
\text { Medium-duty }\end{array}$ & 619.17 \\
& $0.0209 \mathrm{v}_{\mathrm{m}}^{2}$ \\
Heavy-duty & $454.8130-11.8110 \mathrm{v}_{\mathrm{m}}+$ & $1,298.29$ \\
& $0.0878 \mathrm{v}_{\mathrm{m}}^{2}$ & \\
\hline
\end{tabular}

Vehicles available for evacuation can be classified into three types: light-, medium-, and heavy-duty ones. Available numbers, passenger capacities, and equivalent conversion coefficients of the three vehicle types are given in Table 2. Emission factors (running and idle statuses) of these three types of vehicles are displayed in Table 3 . The numbers of evacuees to destinations 1,2 , and 3 are 3155,3600 , and 2700 persons, respectively. The delay time at traffic lights 1 and 2 would be 0.03 and $0.04 \mathrm{hrs}$, respectively. The delay time at the toll station is $0.1 \mathrm{hrs}$. The most and least desirable total evacuation time is 3000 and $6000 \mathrm{hrs}$, respectively. The maximum allowable $\mathrm{CO}$ emission amount is 2.75 to $3.30 \times 10^{5} \mathrm{~g}$ (i.e., $[2.75,3.30] \times 10^{5} \mathrm{~g}$ ). The total budget is approximately $\$ 2.45 \times 10^{4}$ (i.e., $\$ 2.45 \times 10^{4}$ ). Unit operating costs of the ve- 
hicles are highly dependent on vehicle types, manufacturers, and model years, which are all uncertain in nature and obtained as fuzzy sets. The costs of the three vehicle types corresponding to varied $\alpha$-cut levels are provided in Table 4 .

Table 4. Unit Operating Costs of Vehicles under Different $\alpha$-cut Levels $(\$ / \mathrm{km})$

\begin{tabular}{llll}
\hline$\alpha$ & $\begin{array}{l}\text { Light-duty } \\
\left(F_{\sim}^{ \pm}\right)\end{array}$ & $\begin{array}{l}\text { Medium-duty } \\
\left(F_{\sim 2}^{ \pm}\right)\end{array}$ & $\begin{array}{l}\text { Heavy-duty } \\
\left(F_{\sim 3}^{ \pm}\right)\end{array}$ \\
\hline 0 & {$[2.90,3.10]$} & {$[4.90,5.10]$} & {$[7.10,7.30]$} \\
0.2 & {$[2.92,3.08]$} & {$[4.92,5.08]$} & {$[7.12,7.28]$} \\
0.5 & {$[2.95,3.05]$} & {$[4.95,5.05]$} & {$[7.15,7.25]$} \\
0.8 & {$[2.98,3.02]$} & {$[4.98,5.02]$} & {$[7.18,7.22]$} \\
1 & 3.00 & 5.00 & 7.20 \\
\hline
\end{tabular}

This problem calls for efficient evacuation of the evacuees through limited transportation resources under restrictions of economic cost and environmental emission. At the stage of evacuation planning, two critical issues need to be addressed: (i) which are the best routes to take; and (ii) which types of vehicles are to be employed and how to allocate them under a number of restrictions. In this case, many system components as well as their interactions are subject to fuzziness, representing a major challenge confronting the decision makers. Simple decision processes by direct analysis/assessment or expert consultation would not be sufficient for effecttively reflecting the complex system characteristics. A theoretically sound and practically viable way to address this planning problem is to employ the proposed emoFEM model for robust decision support.

The evacuation schemes generated by the emoFEM model are illustrated in Figures 1 to 3. For reaching destination 1, there are two paths available, including path 11 which consists of links 4 and 6 and path 12 which consists of links 2 and 7 . As shown in Figure 1, most of the vehicles would be allocated to path 12 instead of path 11 . In detail, on path 11 , there would be only one light-duty vehicle. Neither medium-duty nor heavy-duty ones would be designated to this path. On path 12, a total of 53 vehicles would be used, including 1 light-duty, 0 medium-duty, and 52 heavy-duty ones. Although the length of path $12(10.1 \mathrm{~km})$ is slightly longer than that of path $11(10$ $\mathrm{km}$ ), for the same vehicle type, the travel time and released contaminants on path 12 would be less than those on path 11 . This may be the reason why path 12 would become the primary channel for transporting the evacuees to destination 1 . The total number of vehicles used for transporting 3155 evacuees to destination 1 would be 54. Among these vehicles, 52 vehicles would be heavy-duty, and 1 would be light-duty. None of the medium-duty vehicles would be employed for reaching evacuation 1.

For destination 2, the path could be path 21 (links 4 and 5), 22 (links 4, 6 and 8), or 23 (links 2, 7 and 8). Path 21, 22, and 23 would have 27,101 , and 30 evacuation vehicles, respectively (Figure 2). Path 22 is thus the dominating evacuation path for destination 2. Of the 27 vehicles on path 21,26 would be medium-duty ones, while only 1 would be light-du-

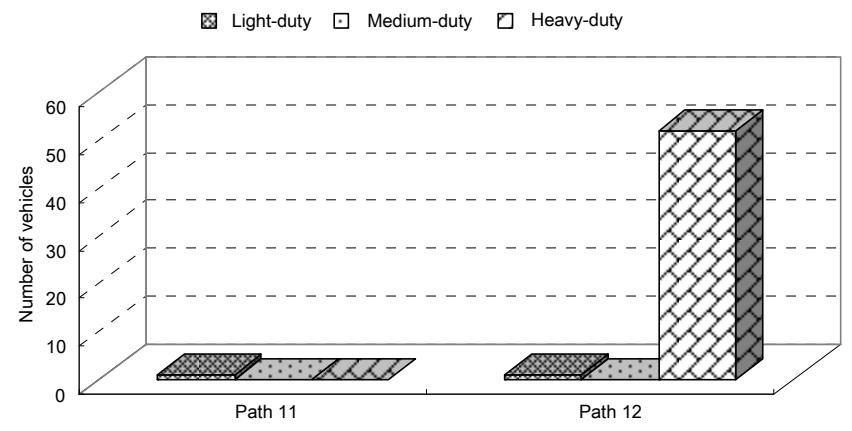

Figure 1. Evacuation scheme for destination 1.

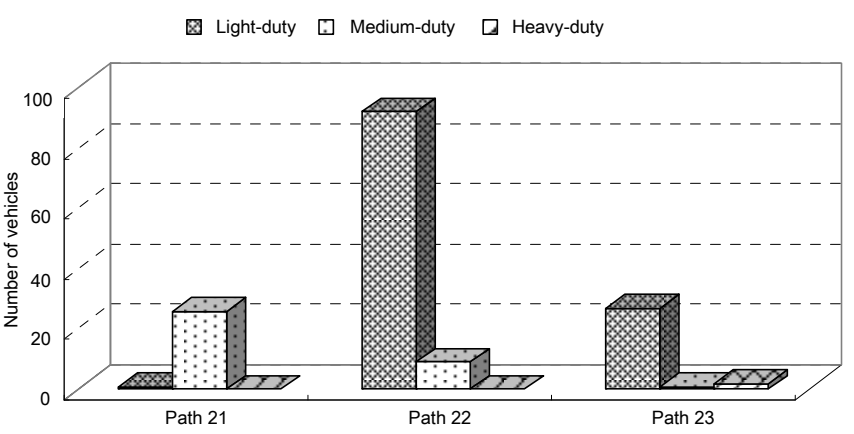

Figure 2. Evacuation scheme for destination 2.

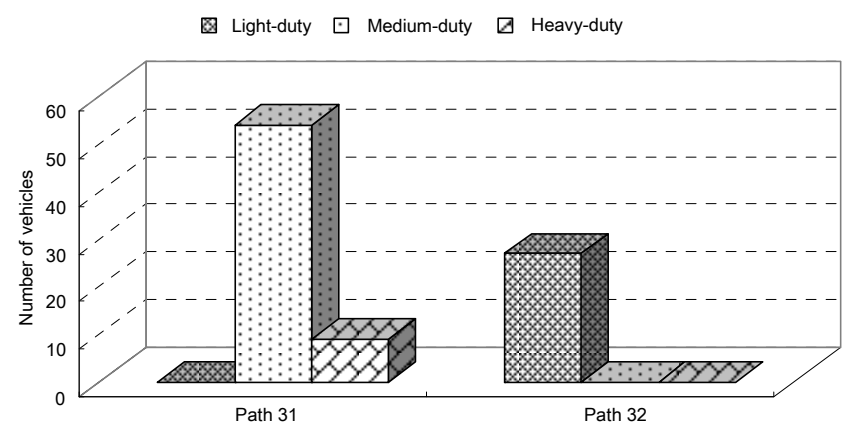

Figure 3. Evacuation scheme for destination 3.

ty. Heavy-duty vehicles would not be used on this path. On path 22, 92 light-duty and 9 medium-duty vehicles would be allocated. Similar to path 21 , path 22 would not use heavyduty vehicles either. On path 23, 27 light-duty, 1 mediumduty, and 2 heavy-duty vehicles would be employed. In total, 158 vehicles would be employed for evacuating 3600 event participants to destination 2, including 120 light-duty, 145 medium-duty, and 2 heavy-duty ones. Thus, heavy-duty vehicles would barely be used for destination 2. Comparatively, medium-duty vehicles would be heavily used, followed by light-duty ones.

For destination 3, the evacuees would be transported via path 31 or 32 . Path 31 includes only one link (i.e., link 1), and path 32 is comprised of two links (i.e., links 2 and 3). As illustrated in Figure 3, path 31 would be the primary path for the evacuees to reach destination 3. This is because that, among the vehicles designated to destination 3, 63 would take path 31 , while 27 would take path 32 . On path 31 , most of the 
(a) Light-duty vehicles

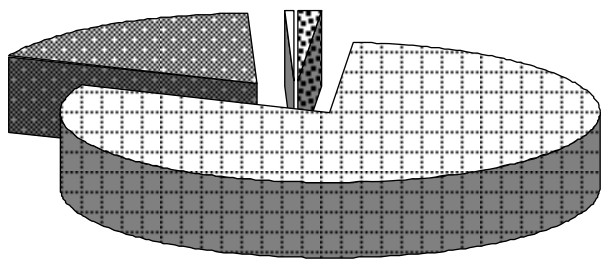

(b) Medium-duty vehicles

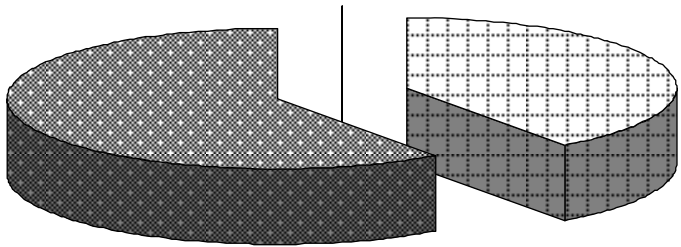

(c) Heavy-duty vehicles

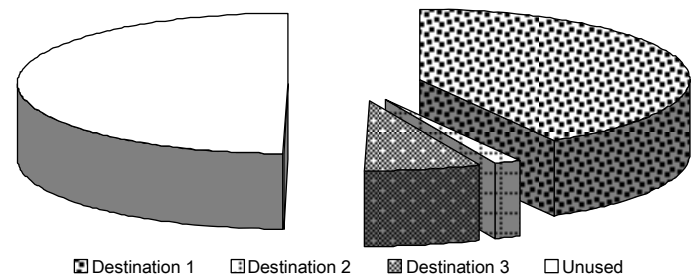

Figure 4. Utilization of the three types of vehicles: (a) lightduty vehicles; (b) medium-duty vehicles; and (c) heavy-duty vehicles.

employed vehicles (i.e., 54) would be medium-duty ones. Besides the medium-duty ones, 9 heavy-duty vehicles would also be used on this path. For path 32, all of the vehicles (i.e., 27 ) would be light-duty ones. None of the medium- and heavy-duty ones would be selected. Totally, 90 vehicles would be used to evacuate 2700 event participants to destination 3 . Among these vehicles, 27 would be light-duty, 54 would be medium-duty, and 9 would be heavy-duty.

All of the 7 available paths would be selected during the process of evacuation. This could be attributed to a number of facts. To new a few, the number of evacuees is large, the normal traffic flows on most of the road links are not heavy, and the requirements for air quality and emission control are stringent. In terms of vehicle selection, decision makers would prefer to employ medium-duty vehicles. This is concluded from the result that all of the 90 medium-duty vehicles would be utilized for evacuation. Light-duty vehicles would also be favored by decision makers, since that 149 of the 150 available light-duty vehicles would be used. In contrast, heavyduty vehicles would be the last choice. Specifically, of the 125 available heavy-duty ones, only 63 would be utilized. This is because that, despite of its largest loading capacity, a heavyduty one possesses the highest unit operating cost and the poorest emission performance among these three vehicle types. Among the 149 light-duty vehicles utilized during the evacuation process, almost all of them (i.e., 120) would be allocated to destination 2, while a small portion would go to
Table 5. Comparisons of Solutions from emoFEM and emoEM

\begin{tabular}{|c|c|c|c|c|}
\hline \multirow{2}{*}{ Destination } & \multirow{2}{*}{ Path } & \multirow{2}{*}{ Vehicle i } & \multicolumn{2}{|c|}{ Solution } \\
\hline & & & emoFEM & emoEM \\
\hline \multirow[t]{6}{*}{1} & \multirow[t]{3}{*}{1} & Light-duty & 1 & 26 \\
\hline & & Medium-duty & 0 & 87 \\
\hline & & High-duty & 0 & 0 \\
\hline & \multirow[t]{3}{*}{2} & Light-duty & 1 & 0 \\
\hline & & Medium-duty & 0 & 1 \\
\hline & & High-duty & 52 & 0 \\
\hline \multirow[t]{9}{*}{2} & \multirow[t]{3}{*}{1} & Light-duty & 1 & 1 \\
\hline & & Medium-duty & 26 & 0 \\
\hline & & High-duty & 0 & 0 \\
\hline & \multirow[t]{3}{*}{2} & Light-duty & 92 & 5 \\
\hline & & Medium-duty & 9 & 0 \\
\hline & & High-duty & 0 & 2 \\
\hline & \multirow[t]{3}{*}{3} & Light-duty & 27 & 0 \\
\hline & & Medium-duty & 1 & 0 \\
\hline & & High-duty & 2 & 56 \\
\hline \multirow[t]{6}{*}{3} & \multirow[t]{3}{*}{1} & Light-duty & 0 & 67 \\
\hline & & Medium-duty & 54 & 2 \\
\hline & & High-duty & 9 & 5 \\
\hline & \multirow[t]{3}{*}{2} & Light-duty & 27 & 50 \\
\hline & & Medium-duty & 0 & 0 \\
\hline & & High-duty & 0 & 0 \\
\hline Total evacua & e (hrs) & & 3789.1 & 3722.9 \\
\hline
\end{tabular}

destinations 1 and 3 (i.e., 2 light-duty and 27 heavy-duty for destinations 1 and 3, respectively). Of the 90 medium-duty vehicles, 36 and 54 vehicles would be assigned for destinations 2 and 3, respectively. In comparison, none of them would be used for reaching destination 1 . As for the 63 heavyduty vehicles adopted for evacuation, most of them (i.e., 52) would be designated to destination 1 . Those used for destinations 2 and 3 would be merely 2 and 9, respectively (Figure $4)$.

The solution for $\lambda$ (i.e., the overall satisfaction degree) would be 0.74 . It represents the degree to which the model's solution fulfills the fuzzy goals and constraints. As discussed above, decision makers' aspiration level for the total evacuation time is $[3000,6000] \mathrm{hrs}$. This means that they would not accept a scheme if the evacuation time is greater than 6000 hrs, while they would be totally satisfied if the evacuation time is no more than $3000 \mathrm{hrs}$. Their satisfaction degree would gradually ascend when the evacuation time reduces from 6000 to $3000 \mathrm{hrs}$. Corresponding to the optimized satisfaction degree of 0.74 , the optimal evacuation time would be $3789 \mathrm{hrs}$ in this study. Likewise, the tolerance interval for the maximum $\mathrm{CO}$ emission amount is $[2.75,3.30] \times 10^{5} \mathrm{~g}$, and the optimized amount of emitted $\mathrm{CO}$ would be $2.89 \times 10^{5} \mathrm{~g}$. The proposed emoFEM model could fulfill decision makers' fuzzy management goals, and directly communicate ambiguous information into the optimization process. Moreover, emoFEM allows moderate constraint violations by relaxing the constraints within tolerance intervals. This would facilitate the analyses of interactions between system optimality and 
system satisfaction. In detail, a lower satisfaction degree corresponds to a higher evacuation time; comparatively, a higher satisfaction degree would suggest reduced evacuation time and improved system efficiency. This feature makes it superior to a deterministic model because the latter one would be simply infeasible when any of the constraints is violated.

To demonstrate the effectiveness of emoFEM, its solutions are compared to those obtained from the emoEM model. In emoEM, all of the fuzzy sets are simplified into crisp (i.e., deterministic) values. As indicated in Table 5, these two models would generate different solutions, and thus provide different vehicle allocation plans and routing strategies. For example, according to emoFEM, path 2 and light-duty vehicles would be the primary path and vehicles for reaching destination 2 , respectively. In comparison, as provided by emoEM, path 3 would be the primary path for destination 2, and most of the employed vehicles on this path would be highduty ones. Such differences in solutions are attributed to the fact that emoFEM could address fuzzy membership functions that are oversimplified in emoEM. In emoEM, only the values with the highest membership grade (i.e., 1) would be used. Thus, emoEM merely represents one of the numerous inputs embedded within the emoFEM model. In other words, emoEM only considers a special case of emoFEM. Moreover, emoFEM could provide system-satisfaction information associated with the obtained solutions, which is beyond the capability of emoEM. Generally, compared to emoEM, emoFEM represents a more realistic reflection of the complexities in event-related evacuation systems, leading to enhanced system robustness.

\section{Conclusions}

This study sought to provide a scientific basis for emission-mitigation-oriented evacuation planning in the face of uncertainty. An emission-mitigation-oriented fuzzy evacuation management (emoFEM) model was proposed to support integrated event-related evacuation management under a fuzzy environment. Besides evacuation efficiency, a number of important factors (e.g., contaminant emission, economic cost and resource availability) were incorporated into the modelling framework, helping identify timely, safe and cost-effective evacuation schemes without significant disturbance over normal municipal traffic and environmental quality. Fuzziness in the model's goals, stipulations and coefficients could be quantified and directly communicated into the optimization process, greatly improving conventional tools for evacuation management under uncertainty.

The results acquired from the application of the developed modelling and solution approach to a hypothetic evacuation network justified its theoretical and practical value. Useful solutions were generated in the case study, helping generating desired vehicle allocation plans and routing strategies with evacuation purposes. Compromising schemes balancing the objectives of higher system efficiency, less contaminant emission, and lower economic cost could be achieved. The results would also support in-depth analyses of interact- tions among system optimality and satisfaction. The advantages of the proposed emo-FEM model were further revealed through comparing its solutions to those from its deterministic counterpart.

Although emoFEM represented an attempt in the field of event-related evacuation planning under uncertainty, its performance could be improved through further efforts. Integration of probability theory into the general framework of emoFEM would be helpful for addressing more complex uncertainties in evacuation management systems. Also, a computeraided decision support system could be advanced through coupling the obtained evacuation plans with practical evacuation operations, in which the emoFEM model could be one of the inexact modelling modules.

Acknowledgments. This research was supported by the National Natural Science Foundation of China (Grant No. 51209087), and the special fund of State Key Lab of Water Environment Simulation (11Z01ESPCN). The authors would like to extend special appreciation to the editor and the anonymous reviewers for their constructive comments and suggestions in improving the quality of this paper.

\section{References}

Birant, D. (2011). Comparison of decision tree algorithms for predictting potential air pollutant emissions with data mining models. $J$. Environ. Inf., 17(1), 46-53. http://dx.doi.org/10.3808/jei.201100186

Cai, Y.P., Huang, G.H., Yang, Z.F., and Tan, Q. (2009). Identification of optimal strategies for energy management systems planning under multiple uncertainties. Appl. Energy, 86(4), 480-495. http://dx. doi. org/10.1016/j.apenergy.2008.09.025

Cai, Y.P., Huang, G.H., Tan, Q., and Chen, B. (2011a). Identification of optimal strategies for improving eco-resilience to floods in ecologically vulnerable regions of a wetland. Ecol. Model., 222(2), 360-369. http://dx.doi.org/10.1016/j.ecolmodel.2009.12.012

Cai, Y.P., Huang, G.H., Tan, Q., and Yang, Z.F. (2011b). An integrated approach for climate-change impact analysis and adaptation planning under multi-level uncertainties. Part I: Methodology. Renew. Sustainable Energy Rev., 15(6), 2779-2790. http://dx.doi.org/10.10 16/j.rser.2011.03.013

Cai, Y.P., Huang, G.H., Tan, Q., and Liu, L. (2011c). An integrated approach for climate-change impact analysis and adaptation planning under multi-level uncertainties. Part II: Case study. Renew. Sustainable Energy Rev., 15(6), 3051-3073. http://dx.doi.org/10. 1016/j.rser.2011.03.014

Chiu, Y.C., Zheng, H., Villalobos, J., and Gautam, B. (2007). Modeling no-notice mass evacuation using a dynamic traffic flow optimization model. IIE Trans., 39(1), 83-94. http://dx.doi.org/10. 1080/07408170600946473

Cova, T.J., and Church, R.L. (1997). Modelling community evacuation vulnerability using GIS. Int. J. Geogr. Inf. Sci., 11(8), 763-784. http://dx.doi.org/10.1080/136588197242077

Dong, C., Tan, Q., Huang, G.H., and Cai, Y.P. (2014). A dual-inexact fuzzy stochastic model for water resources management and nonpoint source pollution mitigation under multiple uncertainties. Hydrol. Earth Syst. Sci., 18(5), 1793-1803. http://dx.doi.org/10. 5194/hess-18-1793-2014

Dubois, D., and Prade, H. (1999). On fuzzy interpolation. Int. J. Gen. Syst., 28(2), 103-112. http://dx.doi.org/10.1080/030810799089352 31

Gokhale, S., and Khare, M. (2007). Statistical behavior of carbon monoxide from vehicular exhausts in urban environments. Environ. 
Model. Software, 22(4), 526-535. http://dx.doi.org/10.1016/j.env soft.2006.02.008

Gorham, R. (2002). Air Pollution from Ground Transportation: An Assessment of Causes, Strategies and Tactics, and Proposed Actions for the International Community, United Nations, New York.

Gunalay, Y., Yeomans, J.S., and Huang, G.H. (2012). Modelling to generate alternative policies in highly uncertain environments: An application to municipal solid waste management planning. $J$. Environ. Inf., 19(2), 58-69.

Hamza-Lup, G.L., Hua, K.A., and Peng, R. (2005). Applying e-transportation to traffic evacuation management under human-caused threats. Proc. of the 2005 IEEE International Conference on ETechnology, E-Commerce and E-Service (EEE'05), Hong Kong, China, March 29-April 1, pp. 666-673. http://dx.doi.org/10.1109/E EE.2005.34

Hobeika, A.G., and Kim, C. (1998). Comparison of traffic assignments in evacuation modeling. IEEE Trans. Eng. Manage., 45(2), 192-198. http://dx.doi.org/10.1109/17.669768

Huang, G.H., Baetz, B.W., and Patry, G.G. (1993). A grey fuzzy linear programming approach for municipal solid waste management planning under uncertainty. Civ. Eng. Syst., 10(2), 123-146. http:// dx. doi.org/10.1080/02630259308970119

Huang, G.H., Cohen, S.J., Yin, Y.Y., and Bass, B. (1996). Incorporation of inexact dynamic optimization with fuzzy relation analysis for integrated climate change impact study. J. Environ. Manage., 48(1), 45-68. http://dx.doi.org/10.1006/jema.1996.0065

Huang, G.H., and Chang, N.B. (2003). The perspectives of environmental informatics and system analysis. J. Environ. Inf., 1(1), 1-6. http://dx.doi.org/10.3808/jei.200300001

Huang, G.H., Sae-Lim, N., Liu, L., and Chen, Z. (2001). An intervalparameter fuzzy-stochastic programming approach for municipal solid waste management and planning. Environ. Model. Assess., 6(4), 271-283. http://dx.doi.org/10.1023/A:10133941188 63

Inuiguchi, M., and Sakawa, M. (1998). Robust optimization under softness in a fuzzy linear programming problem. Int. J. Approximate Reasoning, 18(1-2), 21-34. http://dx.doi.org/10.1016/S0888613X(97)10002-0

Jha, M., Moore, K., and Pashaie, B. (2004). Emergency evacuation planning with microscopic traffic simulation. Trans. Res. Rec., 1886, 40-48. http://dx.doi.org/10.3141/1886-06

Kongtip, P., Thongsuk, W., Yoosook, W., and Chantanakul, S. (2006). Health effects of metropolitan traffic-related air pollutants on street vendors. Atmos. Environ., 40(37), 7138-7145. http://dx.doi.org/10. 1016/j.atmosenv.2006.06.025

Leung, Y. (1988). Spatial Analysis and Planning under Imprecision, North-Holland, Amsterdam.

Li, T.Z. (2001). Study on Evaluation and Forecast Technology of Urban Traffic Atmospheric Environment Influence, Ph.D. Dissertation, Southeast University, Nanjing, Jiangsu, China.

Lin, P., Lo, S.M., Huang, H.C., and Yuen, K.K. (2008). On the use of multi-stage time-varying quickest time approach for optimization of evacuation planning. Fire Saf. J., 43(4), 282-290. http://dx.doi. org/10.1016/j.firesaf.2007.08.005

Liu, L., Huang, G.H., Liu, Y., and Fuller, G.A. (2003). A fuzzystochastic robust programming model for regional air quality management under uncertainty. Eng. Optimiz., 35(2), 177-199. http://dx.doi.org/10.1080/0305215031000097068

Liu, Y., Lai, X.R., and Chang, G. L. (2006). Two-level integrated optimization system for planning of emergency evacuation. J. Transp.
Eng., 132(10), 800-807. http://dx.doi.org/10.1061/(ASCE)0733-94 7X (2006)132:10(800)

Luhandjula, M.K., and Gupta, M.M. (1996). On fuzzy stochastic optimization. Fuzzy Sets Syst., 81(1), 47-55. http://dx.doi.org/10. 1016/0165-0114(95)00240-5

Read, C. (1994). How Vehicle Pollution Affects Our Health, Ashden Trust, London.

Singer, B.C., and Harley, R.A. (1996). Fuel-based motor vehicle emission inventory. J. Air Waste Manage. Assoc., 46(6), 581-593. http://dx.doi.org/10.1080/10473289.1996.10467492

Soyster, A.L. (1973). Convex programming with set-inclusive constraints: Applications to inexact linear programming. Oper. Res., 21(5), 1154-1157. http://dx.doi.org/10.1287/opre.21.5.1154

Tan, Q., Huang, G.H., and Cai, Y.P. (2010a). A superiority-inferioritybased inexact fuzzy stochastic programming approach for solid waste management under uncertainty. Environ. Model. Assess., 15(5), 381-396. http://dx.doi.org/10.1007/s10666-009-9214-6

Tan, Q., Huang, G.H., and Cai, Y.P. (2010b). Radial-interval linear programming for environmental management under varied protecttion levels. J. Air Waste Manage. Assoc., 60(9), 1078-1093. http:// dx.doi.org/10.3155/1047-3289.60.9.1078

Tan, Q., Huang, G.H., and Cai, Y.P. (2011a). Radial interval chanceconstrained programming for agricultural non-point source water pollution control under uncertainty. Agric. Water Manage., 98(10), 1595-1606. http://dx.doi.org/10.1016/j.agwat.2011.05.013

Tan, Q., Huang, G.H., and Cai, Y.P. (2011b). Robust planning of environmental management systems with adjustable conservativeness under compound uncertainty. J. Environ. Eng. - ASCE, 138(2), 208-222. http://dx.doi.org/10.1061/(ASCE)EE.1943-7870.0000469

Tan, Q., Huang, G.H., and Cai, Y.P. (2013). Multi-source multi-sector sustainable water supply under multiple uncertainties: an inexact fuzzy-stochastic quadratic programming approach. Water Resour. Manage., 27(2), 451-473. http://dx.doi.org/10.1007/s11269-012-01 96-9

Urbanik, T. (1978). Texas Hurricane Evacuation Study, The Coastal and Marine Council, Austin, Texas.

Wilmot, C.G., and Mei, B. (2004). Comparison of alternative trip generation models for hurricane evacuation. Nat. Hazards Rev., 5(4), 170-178. http://dx.doi.org/10.1061/(ASCE)1527-6988 (2004) 5:4(170)

Wu, C.Z., Huang, G.H., Yan, X.P., Cai, Y.P., Li, Y.P., and Lv, N.C. (2009). An inexact optimization model for evacuation planning. Kybernetes, 38(10), 1676-1683. http://dx.doi.org/10.1108/0368492 0910994033

Xie, C., Lin, D.Y., and Waller, S.T. (2010). A dynamic evacuation network optimization problem with lane reversal and crossing elimination strategies. Transp. Res. E: Logistics Transp. Rev., 46(3), 295-316. http://dx.doi.org/10.1016/j.tre.2009.11.004

Yi, W., and Ozdamar, L. (2007). A dynamic logistics coordination model for evacuation and support in disaster response activities. Eur. J. Oper. Res., 179(3), 1179-1193. http://dx.doi.org/10.1016/j. ejor.2005.03.077

Yoshida, Y., and Matsuhashi, R. (2009). Evaluation of $\mathrm{CO}_{2}$ emission reduction in Japan utilizing the interregional repercussion model on freight transportation. J. Environ. Inf., 14(1), 41-50. http://dx. doi.org/10.3808/jei.200900152

Zimmermann, H.J. (2001). Fuzzy Set Theory and Its Applications, 4th edition, Kluwer Academic Publishers, Boston. http://dx.doi.org/10. 1007/978-94-010-0646-0 